\title{
Effects of nitric oxide on sGC redox states
}

\section{Martin Deile*1, César Ibarra-Alvardo1, Pavel I Nedvetsky ${ }^{1}$, Helmut Müller ${ }^{1}$, Barbara Kemp-Harper ${ }^{3}$, Johannes-Peter Stasch ${ }^{2}$ and HHHW Schmidt ${ }^{1,3}$}

\author{
Address: ${ }^{1}$ Rudolf-Buchheim-Institute for Pharmacology, University of Giessen, 35392 Giessen, Germany, ${ }^{2}$ Cardiovascular Research, Bayer \\ HealthCare, 42096, Wuppertal, Germany and ${ }^{3}$ Department of Pharmacology, Monash Universiy, Melbourne, VIC 3800, Australia \\ Email: Martin Deile* - martin.deile@med.uni-giessen.de \\ * Corresponding author
}

from 2nd International Conference of cGMP Generators, Effectors and Therapeutic Implications

Potsdam, Germany, 10-12 June, 2005

Published: 16 June 2005

BMC Pharmacology 2005, 5(SuppI I):PI3 doi:I0.II86/I47I-22I0-5-SI-PI3

Soluble guanylyl cyclase (sGC), the best-established nitric oxide receptor is a heterodimeric heme-protein synthesizing cGMP upon NO binding to its $\mathrm{Fe}(\mathrm{II})$ heme. cGMP in turn plays an important role in the regulation of vascular tone and platelet aggregation. The redox state of the sGC heme is fundamental for the activation by NO. A novel class of sGC activators, such as the recently described BAY 58-2667, are however NO-independent and target sGC in its $\mathrm{Fe}(\mathrm{III})$ and heme-free state. Given the therapeutic potential of such sGC activators, the mechanisms that modulate the sensitivity of sGC towards these agonists is of great interest. In this study we found, that treatment of primary porcine pulmonary artery endothelial cells with the NO donor DETA-NO $(100 \mu \mathrm{mol} / \mathrm{l})$ leads to a downregulation of DEA-NO stimulated and an up-regulation of BAY 58-2667 stimulated sGC activities ( $\mathrm{pmol} / 10^{6}$ cells) [DEA-NO: $4.83 \pm 0.11$ vs. $3.02 \pm 0.08^{* * *}$, BAY 58-2667: $0.77 \pm 0.01$ vs. $1.91 \pm 0.04^{* * *}$; control vs. 2 h DETA-NO pre-treatment]. Furthermore, we observed a down-regulation of sGC $(\alpha 1 / \beta 1)$ protein levels upon treatment with DETA-NO in a dose- and time-dependent manner. Interestingly, the decrease in protein levels (maximal at $\mathrm{t}=72$ h: $56.7 \pm 7 \%$ of control for sGC $\alpha 1$ and $44.4 \pm 8 \%$ of control for sGC $\beta 1$ ) occured subsequent to the apparent reduction in NO stimulatable $\mathrm{sGC}$ activity (maximal at $\mathrm{t}=$ $2 \mathrm{~h}$ ). Downregulation of cellular cGMP responses has recently been attributed to upregulation of cGMP breakdown via enhanced PDE5 activity. Thus we tested the effects of the cGMP analogue 8-Br-cGMP (125/250/500 $\mu \mathrm{mol} / \mathrm{l})$, the mixed NO-independent sGC activator and PDE inhibitor YC-1 (10/50/100 $\mu \mathrm{mol} / \mathrm{l})$ and the selective phosphodiesterase-5 inhibitor zaprinast (50/100/200 $\mu \mathrm{mol} / \mathrm{l}$ ) on sGC protein levels. In contrast to DETA-NO, none of these modulators altered sGC protein expression arguing against a cGMP/PDE dependent mechanism. To determine if endogenously produced $\mathrm{NO}$ mimicked the effects of DETA-NO on sGC we utilised two models. First, porcine endothelial cells were pretreated with the endothelial NO synthase (eNOS) inhibitor L-NAME (100 $\mu \mathrm{mol} / \mathrm{l}$ from 2 to 72 hours) and, second, aortas from eNOS deficient mice were investigated. L-NAME induced changes in endothelial NO and BAY 58-2667 stimulated sGC activity, that were reciprocal to those observed with DETA-NO treatment. While sGC expression and the response to DEA-NO were enhanced, the response to BAY 58-2667 was un-altered. Concurrent to these findings, in aortic homogenates from eNOS -/- mice, an increased DEA-NO induced sGC response was observed as compared to wild type controls. In conclusion, our data suggests endogenous regulation of the sGC redox state via NO. The decrease in the cGMP response to DEA-NO and the increase to BAY 58-2667 induced cGMP production following DETA-NO pretreatment, indicates a change in the redox state of the sGC heme from a ferrous to ferric or even heme free. Furthermore, these changes in the redox state of sGC, seem to induce a protein degradation of sGC ( $\alpha 1 / \beta 1)$. Hence, desensitization of the NO/cGMP-pathway is heterogenous, occurring either as a result of PDE-5 upregulation or, as in pulmonary endothelial cells, via a PDE-5-independent mechanism involving a change in sGC redox state and protein stability. 